CORRECTION

\title{
Correction to: Study on the Effect of the Grain Refinement on Mechanical Properties of the P92 Welded Joint
}

C. Pandey, Naveen Kumar, S. Sirohi, T. Rajasekaran, S. Kumar, and P. Kumar

\section{Correction to: \\ JMEPEG \\ https://doi.org/10.1007/s11665-021-06536-z}

In the originally published article, the affiliation for S. Sirohi and S. Kumar was incorrect. The correct affiliation is shown below:
S. Sirohi and S. Kumar, Department of Mechanical Engineering, SRM Institute of Science and Technology, Delhi NCR Campus, Modinagar 201204, Uttar Pradesh, India

The original article has been updated to correct the error.

Publisher's Note Springer Nature remains neutral with regard to jurisdictional claims in published maps and institutional affiliations.

The original article can be found online at https://doi.org/10.1007/ s11665-021-06536-z.

C. Pandey, Mechanical Department, Indian Institute of Technology Jodhpur, N.H. 62, Nagaur Road, Karwar, Jodhpur, Rajasthan 342037, India; Naveen Kumar, Institute of Engineering and Technology, Lucknow 226021, India; S. Sirohi and S. Kumar, Department of Mechanical Engineering, Delhi NCR Campus, SRM Institute of Science and Technology, Uttar Pradesh, Modinagar 201204, India; T. Rajasekaran, Department of Mechanical Engineering, SRM Institute of Science and Technology, Tamil Nadu, Kattankulathur 603203, India; P. Kumar, Department of Production Engineering, National Institute of Technology, Tiruchirappalli, Tamil Nadu 620015, India. Contact e-mails: chandanpy.1989@gmail.com, jscpandey@iitj.ac.in, sachinsirohi2008@gmail.com. 